\title{
Intensivmedizinische Therapie infektiöser Komplikationen in der pädiatrischen Hämatologie und Onkologie
}

\section{Bind ${ }^{1}$ \\ T. Nicolai ${ }^{2}$}

\author{
Management of Septic Shock and Acquired Respiratory Distress Syndrome \\ in Pediatric Cancer Patients
}

\section{Zusammenfassung}

Zum septischen Schock kommt es bei 6\% der pädiatrischen hämatologisch-onkologischen Patienten mit Granulozytopenie und Fieber, mit einer Sepsisletalität von 5\% ohne und 40\% mit Knochemarktransplantation (KMT). Unter allen Kindern mit ARDS leidet ein Drittel an Störungen der Immunkompetenz. Die Letalität beträgt hier ca. $45 \%$, nach KMT ca. $80 \%$. Für den septischen Schock sind zu mehr als 75\% gramnegative Keime ursächlich. Das ARDS ist in über $50 \%$ der Fälle Folge von Pneumonien, darunter $20 \%$ durch opportunistische Erreger und einer Sepsis in $25 \%$. Die vorliegende Arbeit enthält umfassende Empfehlungen der der Arbeitsgruppe „Infektionen bei immunsupprimierten Kindern“ der Deutschen Gesellschaft für Pädiatrische Infektiologie (DGPI) und der Deutschen Gesellschaft für Pädiatrische Onkologie und Hämatologie (GPOH) zur Behandlung bei septischen Schock und akutem Lungenversagen. Therapiemaßnahmen beim septischen Schock sind eine frühe Antibiotikatherapie bzw. Elimination des Herdes und die rasche Volumenexpansion ( $\geq 40 \mathrm{ml} / \mathrm{kg}$ in der ersten Stunde). Der Volumenrefraktäre Schock erfordert den Einsatz von Vasopressoren (Noradrenalin), gefolgt von einer differenzierten Kreislauftherapie. Die Substitution von Hydrokortison ist häufig indiziert. Grundpfeiler der ARDS-Behandlung sind die Therapie des Auslösers und eine Beatmung mit ausreichendem endexspiratorischem Druck (PEEP) und begrenzten Tidalvolumen $(\leq 6 \mathrm{ml} / \mathrm{kg}$ ) bzw. limitierten Spitzendrücken $\left(<35 \mathrm{cmH}_{2} \mathrm{O}\right)$ um eine weitere iatrogene Lungenschädigung zu minimieren. Die Volumentherapie zielt auf eine ausreichende Vorlast, um auch unter Beatmung mit hohem PEEP ein ausrei-

\section{Abstract}

Septic shock occurs in $6 \%$ of paediatric cancer patients with neutropenia and fever. The mortality of the septic shock is $40 \%$ in BMT patients and $5 \%$ in others. One third of paediatric ARDS cases affect immunocompromised individuals with a total mortality of $45 \%$ and $80 \%$ after BMT. Septic shock is caused by gramnegative bacteria in more than $75 \%$. ARDS is due to pneumonia in more than $50 \%$, sepsis in about $25 \%$. This article provides the recommendations of the Infectious Diseases Working Party of the German Society for Pediatric Infectious Diseases (DGPI) and the German Society for Pediatric Hematology/Oncology (GPOH) for treatment of septic shock and ARDS. Therapy of septic shock includes early antibiotic therapy and volume expansion ( $\geq 40 \mathrm{ml} / \mathrm{kg}$ initially). Refractory shock requires vasopressors (noradrenaline), followed by a judicious circulatory management. Hydrocortison is indicated in patients with high probability of adrenal insufficiency. Mainstay of ARDS therapy is ventilation with sufficient endexspiratory pressure (PEEP) to prevent loss of functional residual capacity and with limited tidal volumes $(\leq 6 \mathrm{ml} / \mathrm{kg})$ and limited inspiratory pressure $\left(<35 \mathrm{cmH}_{2} \mathrm{O}\right)$ respectively, to minimize ventilator induced lung injury. Volume therapy consists of maintenance of sufficient preload to counteract the impaired venous return, induced by positive pressure ventilation. Diuretics and eventually veno-venous haemofiltration are used to reduce free lung water. Surfactant application may be considered in severe cases. Steroids are indicated in pneumocystis carinii pneumonia and in engraftment pneumonitis.

Institutsangaben

${ }^{1}$ Univ.-Klinik für Kinderkardiologie, Aachen

${ }^{2}$ Dr. v. Haunersches Kinderspital der LMU, München

Korrespondenzadresse

PD Dr. Lutz Bindl · Klinik f. Kinderkardiologie · RWTH Aachen · Pauwelsstr. 30 • 52057 Aachen ·

Tel.: 02 41/8088986·E-mail: lbindl@ukaachen.de

Bibliografie

Klin Pädiatr 2005; 217 (Suppl 1): S130-S142 • (c) Georg Thieme Verlag KG Stuttgart • New York DOI $10.1055 / \mathrm{s}-2005-872507$

ISSN 0300-8630 
chendes Herzzeitvolumen zu erhalten. Das freie Lungenwasser wird durch Gabe von Diuretika und nötigenfalls eine venovenöse Hämofiltration gesenkt. Die Gabe von Surfactant ist in refraktären Fällen zu erwägen. Steroide sind bei Pneumozystis-Infektionen und beim Engraftment-Syndrom indiziert.

\section{Schlüsselwörter}

Septischer Schock · ARDS · Immundefizienz

\section{Key words}

Septic shock · ARDS · immunodeficiency

\section{Septischer Schock}

\section{Allgemeines (Ursache, Definition, Prognose)}

Es handelt sich beim septischen Schock um die schwerste Form einer systemischen Entzündungs-Reaktion (SIRS) des Körpers auf generalisierte Infektionen (Bakteriämie, Fungämie, Virämie, Parasitämie oder schwere Organinfektion). Je nach dem Schweregrad unterteilt man diese Reaktion des Körpers formal in SIRS (systemische entzündliche Reaktion), Sepsis, schwere Sepsis, septischen Schock und Multiorganversagen.

Prinzipiell unterscheidet sich diese Reaktion bei Kindern nicht von der bei Erwachsenen beobachteten Form, die klinischen Erscheinungsbilder sind jedoch bei Kindern mit onkologischen Erkrankungen anders als bei primär gesunden Kindern. Bestimmte Erregergruppen dominieren als Auslöser in verschiedenen Altersstufen bzw. bei verschiedenen Grunderkrankungen (z. B. Leukämie, Z.n. KMT) und klinischen Situationen (Zeitfenster nach KMT, Leukopenie). Einzelne chronische Vorerkrankungen wie eine koronare Herzerkrankung, die bei der Therapie Erwachsener besondere Probleme bereiten, kommen bei Kindern praktisch nicht vor, während andererseits die adrenergen Kompensationsmechanismen begrenzt sind und der Krankheitsverlauf bei Kindern foudroyanter sein kann.

Aledo et al. 1998 [2] berichten eine Inzidenz des septischen Schocks bei Kindern mit hämatologisch-onkologischer Grunderkrankung von 6,4\% der Aufnahmen wegen Granulozytopenie und Fieber bzw. 19,3\% der Fälle mit positiver Blutkultur. Ca. $30 \%$ der Isolate der Sepsispatienten waren koagulasenegative Staphylokokken, unter den Schockpatienten wurden jedoch in weniger als $25 \%$ der Fälle grampositive Keime isoliert.

Die Immunsuppression durch Chemotherapie bzw. im Rahmen der Grunderkrankung und die zahlreichen möglichen Eintrittspforten (geschädigte Schleimhäute, ggf. bestehende permanente vaskuläre Zugänge wie Portsysteme und Hickman-Katheter) prädisponieren Kinder mit hämatologisch-onkologischen Erkrankungen zur Entwicklung septischer Komplikationen. Die Rolle genetischer Risikofaktoren, die für das Auftreten des septischen Schocks identifiziert wurden (männliches Geschlecht bei Neugeborenen und Säuglingen [16, 76], Polymorphismen der immunmodulatorischen Signalübertragung wie z.B: TLR, CD14, IL-6, IL-10, TNF- $\alpha$ und Proteinen der humoralen Abwehr (z. B. Mannose Binding Lectin [MBL], BPI) und von Gerinnungsfaktoren (z. B. Plasminogen-Aktivator-Inhibitor [PAI]) ist bei diesen Patienten nicht untersucht und vermutlich im Vergleich zu den offensichtlichen, genannten Risikofaktoren von untergeordneter Bedeutung.
Im Gegensatz zu den hohen Letalitätsziffern des septischen Schockes noch zu Beginn der 90er-Jahre ist in den letzten Jahren eine deutliche Besserung eingetreten. Während von Han et al. 2003 unizentrische Daten aus den Jahren 1993-2001 noch eine Gesamtletalität von $29 \%(n=89)$ ausweisen [41], wurde an der Cornell-University im Zeitraum 1998-2001 bei onkologischen Patienten ohne Knochenmarktransplantation eine Letalität der Sepsis von 5,5\%, bei KMT-Patienten von $42,9 \%$ berichtet [4]. Die Erfordernis von Katecholaminen war nur bei Bedarf von mehr als einer Substanz mit erhöhter Letalität (42,9\%) verbunden. Die Letalitätsziffern bei Kindern liegen somit sowohl bei immunsupprimierten Patienten wie auch in gemischten Kollektiven deutlich unter den von Erwachsenen. Dennoch sterben mehr Kinder im Zusammenhang mit einer schweren Sepsis als an onkologischen Erkrankungen [76].

\section{Klinisches Bild}

Formal wird es als SIRS definiert, wenn zwei oder mehr der folgenden Symptome vorliegen: veränderte Temperatur, Tachypnö, Tachykardie, abnormale Leukozytenzahlen (Tab.1). Eine Sepsis wird durch das Vorliegen eines SIRS mit vermuteter oder bewiesener mikrobieller Ätiologie definiert, eine schwere Sepsis durch zusätzliche Dysfunktion eines Organsystems. Der septische Schock ist laut CDC-Definition beim Vorliegen einer arteriellen Hypotonie (systolischer Blutdruck um mehr als 2 SD unter der altersentsprechenden Norm, trotz adäquater Volumengabe oder Vasopressor-Medikation, s. Tab.1) und der Dysfunktion mehrere Organsysteme bei Sepsis gegeben. Die häufig verwendete Gleichsetzung von Hypotension und Schock ist stark vergröbernd, da ein niedriger Blutdruck nicht gleichbedeutend mit einer mangelhaften Systemdurchblutung ist, die über ein zu geringes globales oder regionales Sauerstoffangebot neben dem primär entzündlichen Gewebsschaden eine zusätzliche hypoxisch-ischämische Schädigung verursachen kann. Ein erniedrigter arterieller Blutdruck kann sowohl Folge des systemischen Widerstandsverlustes bei hyperdynamem septischen Schock als auch Folge der dekompensierten Myokardfunktion sein, ist dann also eher ein Spätzeichen des bereits manifesten Schocks.

Offensichtliche klinische Zeichen der mangelhaften Systemperfusion sind Störungen des Bewusstseins und der Hautdurchblutung (Rekapillarisierungszeit > 2 Sekunden; Peripher-zentrale Temperaturdifferenz $>4$ Grad Celsius), der Pulsfüllung sowie eine Oligurie $(<1 \mathrm{ml} / \mathrm{kg} / \mathrm{Std}$.). Insbesondere initial oder bei Leukopenie sind die klinischen Zeichen manchmal subtil. Gelegentlich imponiert initial nur ein grau-blasses Hautkolorit als Zeichen der peripheren Vasokonstriktion. In allen Altersstufen können Krampfanfälle auftreten (dies kann auf eine zerebrale Beteiligung hinweisen, die bei bis zu 30\% der Kinder mit Sepsis 
Tab. 1

\begin{tabular}{|c|c|c|}
\hline \multicolumn{3}{|l|}{ Symptome (SIRS) } \\
\hline \multicolumn{3}{|c|}{$\begin{array}{l}\text { - veränderte Körpertemperatur }>38^{\circ} \mathrm{C} \text { oder }<36^{\circ} \mathrm{C} \\
\text { - Tachykardie, Tachypnö } \\
\text { - Leukozytose/Leukopenie }\end{array}$} \\
\hline \multicolumn{3}{|c|}{ Herz- und Atemfrequenz-Normalwerte } \\
\hline Alter & Herzfrequenz & Atemfrequenz \\
\hline$<1$ Monat & $>190 /$ min & $>60 / \min$ \\
\hline 1-12 Monate & $>160 /$ min & $>45 / \min$ \\
\hline 1-2 Jahre & $>140 /$ min & $>40 / \min$ \\
\hline 3-5 Jahre & $>130 /$ min & $>35 / \min$ \\
\hline 6-12 Jahre & $>120 / \mathrm{min}$ & $>30 / \min$ \\
\hline 13-15 Jahre & $>100 /$ min & $>25 / \min$ \\
\hline$>15$ Jahre & $>90 / \min$ & $>20 / \min$ \\
\hline \multicolumn{3}{|c|}{ Blutdruck-Normalwerte } \\
\hline Alter & diastolischer RR & systolischer RR \\
\hline Neugeborenes & $30-48$ & $50-83$ \\
\hline 3 Monate & $37-60$ & $80-110$ \\
\hline 6 Monate & $43-63$ & $80-110$ \\
\hline 1-3 Jahre & $46-79$ & $80-113$ \\
\hline 4-6 Jahre & $47-79$ & $80-115$ \\
\hline 7-10 Jahre & $52-83$ & $83-122$ \\
\hline 11-13 Jahre & $58-88$ & $95-136$ \\
\hline 14-16 Jahre & $55-77$ & $100-127$ \\
\hline \multicolumn{3}{|c|}{ Leukozyten $>12000 / \mathrm{mm}^{3}$ oder $<4000 / \mathrm{mm}^{3}$ oder $>10 \%$ Stabkernig } \\
\hline
\end{tabular}

vorliegt), als Zeichen der Gerinnungsstörung oder einer Vaskulopathie finden sich petechiale Blutungen (diese bereitet bei Kindern mit vorbestehender Thrombopenie differenzialdiagnostische Schwierigkeiten).

\section{Diagnostische Maßnahmen}

Klinisch wird zunächst die Überwachung der Viatlfunktionen wie Kreislauf, Bewusstsein (Glasgow-Coma Scale), Atmung, Urinproduktion etc. erfolgen. Laboruntersuchungen: Blutbild (mit Differenzialblutbild) und „Basischemie“ (Elektrolyte, CRP, Eiweiß, Blutzucker, Kreatinin, Harnstoff, Bilirubin), Blutgasanalyse, Laktat, Albumin, LDH, Quick, PTT, Fibrinogen, Fibrinogenspaltprodukte, AT III, evtl. $\mathrm{NH}_{3}$, Blutkulturen (aerob/anaerob, Pilzkulturen, ggf. seltene Erreger wie Tbc). Urinstatus, spezifisches Gewicht, Urinkultur, Latexagglutinationstests. Lumbalpunktion nach Klinik (Liquorkultur, Gram-Färbung, Latexagglutinationstests), gegebenenfalls wird eine Stuhlkultur sinnvoll sein. Entscheidend ist die Suche nach möglichen Infektionsquellen (Pneumonie, Harnwegsinfektion, Osteomyelitis, Otitis etc.).

Bei Leukozytopenie sollte frühzeitig, z. B. bei fehlender Entfieberung nach $24-48$ Std., ein Thorax-CT erwogen werden, da trotz pulmonaler Beteiligung das konventionelle Röntgenbild normal erscheinen kann. Pulmonale Pilzinfektionen (Aspergillosen) sind oft nur im CT zu erkennen und erfordern eine rasche spezifische Therapie. Auch eine erweiterte Fokussuche mittels AbdomenSonographie oder -CT und ggf. ein NMR oder CT des Schädels sind bei leukozytopenischen und/oder immunkompromittierten
Patienten nicht nur bei entsprechenden Symptomen frühzeitig zu erwägen.

\section{Therapie}

\section{Prinzipien der Therapie}

Bei diesem potenziell sehr rasch lebensbedrohlichen Krankheitsbild ist zunächst die Sicherung der Vitalfunktionen vordringlich (ABC-Regel). Nach Symptombeginn kann es in kürzester Zeit zu einer massiven Hypoxie lebenswichtiger Organe und Übergang in einen therapierefraktären Schock kommen. Infolgedessen ist die schnellste Bekämpfung der Kreislaufinsuffizienz mit symptomatischen und supportiven Maßnahmen entscheidend, hier sind noch mehr als beim Erwachsenen unter Umständen Minuten entscheidend [63].

Die wichtigste Erkenntnis der letzten Zeit liegt darin, dass die Bekämpfung der Hypovolämie durch rasche, nach Bedarf mehrfach wiederholte Bolusinjektionen von je $20 \mathrm{ml} / \mathrm{kg}$ isotoner Lösungen und/oder Kolloide sofort nach der Diagnosestellung (evtl. kombiniert mit Adrenergika, ggf. beides auch im vorklinischen Bereich) mit dem Ziel der Rekompensation der Kreislauffunktion in den ersten 10 Minuten ( -1 Std.) die Prognose entscheidend beeinflussen kann [41]. Dieses aggressive Volumenmanagement hat z. B. die Mortalität der Meningokokkensepsis halbiert [19].

Neben der supportiven Therapie ist die rasche Gabe von wirksamen Antibiotika von entscheidender Bedeutung. Die altersund situationsangepasste empirische Therapie muss oft vor der eindeutigen Sicherung der Diagnose Sepsis beim bloßen Verdacht einsetzen. Dennoch müssen Erregerkulturen (Blutkultur; Liquorkultur nur falls klinisch zulässig) noch vor der Antibiotikagabe gewonnen werden.

\section{Praktisches Vorgehen}

Die derzeit gültigen Empfehlungen aus der Gruppe „Survivinig Sepsis Campaign“ sind in Abb. 1 wiedergegeben.

\section{Sicherung der Vitalfunktionen}

Beatmungsindikationen sind eine zunehmende Bewusstseinstrübung (bei GCS $<8$ zwingend), Sauerstoffsättigungsabfälle und der volumenrefraktäre hypodyname Kreislaufschock. Eine Intubation wird empfohlen wenn nach zwei rasch injizierten Volumenboli (s.u.) der Schock weiter besteht [59]. Ziel der Beatmung ist es, den Sauerstoffverbrauch zu reduzieren und die arterielle Sauerstoffsättigung über $90 \%$ zu halten, bei ausreichender Vorlast ist auch mit einer Besserung des systemischen Blutdruckes durch den bei Beatmung höheren mittleren intrathorakalen Druck zu erhoffen.

Einer akuten Bradykardie trotz ausreichender Ventilation und Oxygenierung liegt oft eine koronare Minderperfusion vor, die durch rechtzeitigen Beginn einer Herzdruckmassage und Vasopressorgabe sowie die sonstigen Maßnahmen der kardiopulmonalen Reanimation reversibel sein kann.

\section{Frühe Therapie des Kreislaufschocks}

Entscheidend ist bei Kindern die Korrektur der fast immer vorhandenen relativen intravasalen Hypovolämie [32] mit dem Ziel 


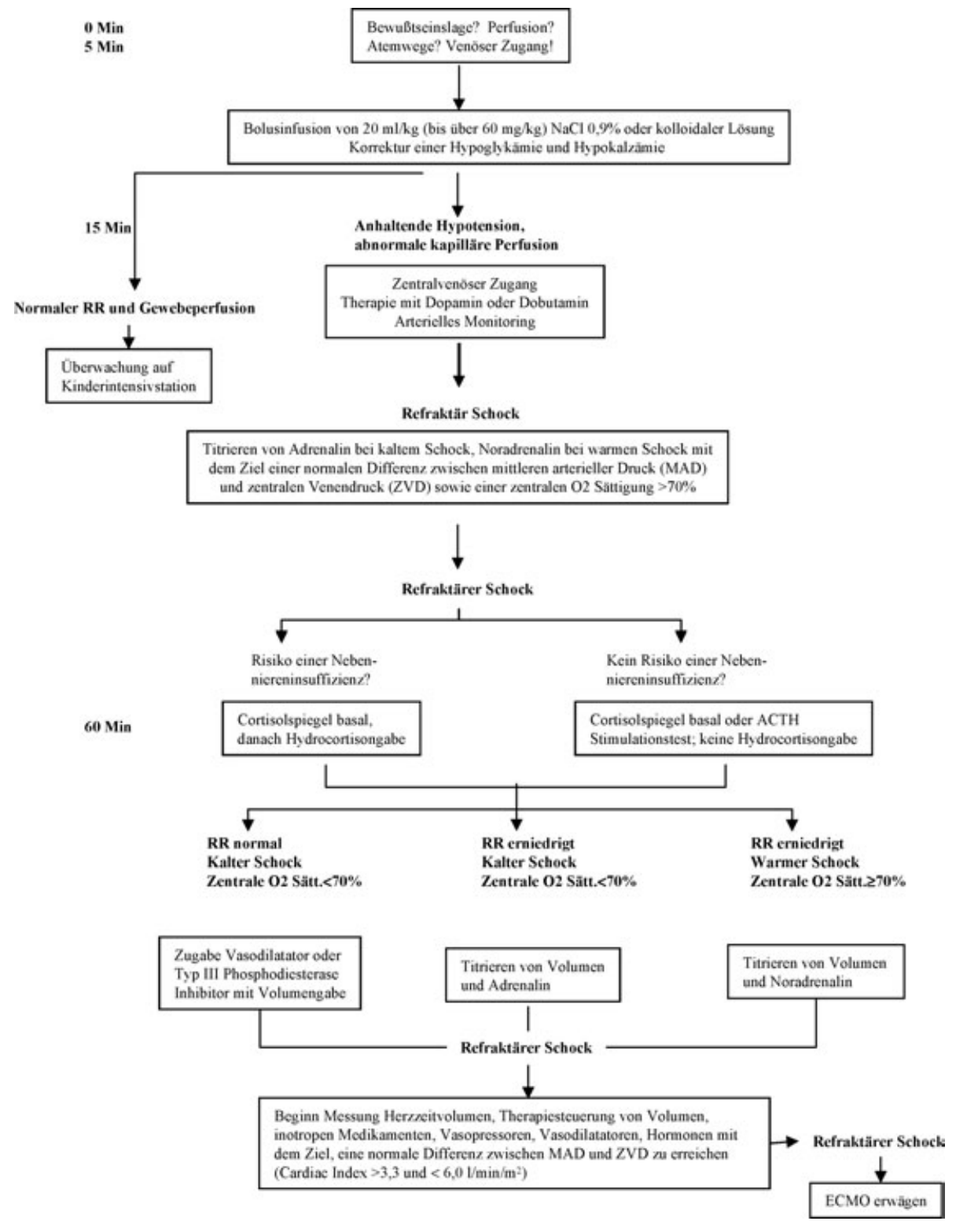

Abb. 1 Flussschema zur Kreislauftherapie des septischen Schocks (in Anlehnung an Carcillo et al. [26]). 
des Erregers die Kombination eines gramnegativ wirksamen Cephalosporines mit einem Aminoglykosid sinnvoll, bei liegendem Katheter wird man ein im gramnegativen Bereich breit wirksames Cephalosporin (z. B. Ceftazidim) mit einem gegen Staphylococcus epidermidis wirksamen Präparat kombinieren. Die infektiologische Eigenanamnese sowie das Erregerspektrum des Behandlungszentrum müssen bei der Auswahl der empirischen Antibiose berücksichtigt werden. Bei vermuteter oder bewiesener Meningitis müssen jeweils die Dosen entsprechend angepasst werden.

Nach Erhalt von positiven mikrobiologischen Kulturen werden die Antibiotika auf das Medikament umgesetzt, das am Ort der Infektion die besten Gewebsspiegel erreicht und gleichzeitig zwar den gefundenen Keim erfasst, jedoch nicht zu breit wirkt. Greift die Therapie klinisch nicht nach $24-48$ Std., so muss das Regime erweitert oder umgesetzt werden, je nach Patient kommen nicht selten Pilz- und Virusinfektionen in Betracht.

\section{Weitere supportive Therapiemaßnahmen}

Es muss auf eine sorgfältige Korrektur von Störungen der Elektrolytwerte geachtet werden. Ein Azidoeseausgleich mit Bikarbonat scheint zumindest bei einem $\mathrm{pH}>$ 7,15 nicht sinnvoll [30]. Insbesondere bei Säuglingen ist die engmaschige Kontrolle des Blutzuckers wegen drohender Hypoglykämien notwendig. Pädiatrische Daten zur strikten Kontrolle einer Hyperglykämie existieren nicht.

\section{Differenzierte Kreislauftherapie Ziel, Monitoring}

Ziel der Therapie ist ein normaler Blutdruck, eine Rekapillarisierungszeit $<2 \mathrm{~s}$, gute periphere Durchblutung mit warmen Extremitäten und eine (gemischtvenöse bzw. möglichst aus der Vena Cava superior abgenommene) venöse $\mathrm{O}_{2}$-Sättigung $>70 \%$. Die Urinausscheidung ( $>1 \mathrm{ml} / \mathrm{kg}$ ) kann als Maß einer ausreichenden Organperfusion herangezogen werden, da die Niere den zweithöchsten Anteil an Perfusion aller Organe erhält und infolge gestörter Autoregulation der Durchblutung im Schock oft eine direkte Abhängigkeit der Urinproduktion vom mittleren Perfusionsdruck besteht [27]. Bei erhöhtem intraabdominalem Druck kann die renale Perfusion sekundär herabgesetzt sein [38].

Lässt sich das Therapieziel bereits durch aggressive Volumengabe alleine erreichen, ist eine invasives Kreislaufmonitoring nicht erforderlich. Beim volumenresistenten Schock ist eine rasche Katecholamingabe erforderlich, und im weiteren Verlauf deshalb ein invasiveres Monitoring nötig. Es wird empfohlen, zur Medikamentengabe und Therapiesteuerung einen zentralvenösen und arteriellen Zugang zu schaffen, um ZVD (Ziel: ca. $12 \mathrm{~cm}$ $\mathrm{H}_{2} \mathrm{O}$ ) und arterielle Drücke (Ziel: altersentsprechend normal) sicher messen zu können.

Die Verwendung eines Pulmonaliskatheters ist nur gerechtfertigt, wenn sich trotz Volumen, differenzierter Katecholamingabe und Therapiesteuerung nach klinischen Parametern, ggf. mit Nachlastsenkern, die therapeutischen Ziele nicht erreichen lassen und als Ursache eine fehlerhafte Einschätzung des peripheren Widerstandes und des Herzzeitvolumens vermutet wird [73]. Dies dürfte nur für eine sehr kleine Minderheit von Patienten zutreffen, und unserer Ansicht nach ist in diesen Fällen eine weniger invasive Messung mittels Indikator-Verdünnungsmethode (PiCCO [,pulse contour continuous cardiac output“]) evtl. erfolgversprechend. Wegen der relativ großen Störanfälligkeit und dem Fehlen von Normal- oder Sollwerten für Kinder ist jedoch eine Bewertung solcher Messungen unseres Erachtens nur im klinischen Kontext und bei großer Erfahrung sinnvoll. Bei Erwachsenen hat sich eine erhöhte Mortalität durch Pulmonaliskatheter für Patienten mit eher mildem Sepsisverlauf zeigen lassen, und einen Nutzen nur bei den schwersten Verläufen [29].

\section{Praktisches Vorgehen}

Wenn der Kreislauf nach Restitution eines ausreichenden intravasalen Volumens (Halsvenen gefüllt, Herz im Röntgen-Thorax nicht klein, Mediastinalbreite groß, evtl. ZVD hochnormal oder hoch) insuffizient bleibt, liegt möglicherweise ein peripherer arterieller Widerstandsverlust oder eine myokardiale Funktionsbeeinträchtigung (sehr häufig bei Kindern) vor. Eine echte hyperdyname Initialphase mit erhöhtem Herzminutenvolumen wie beim Erwachsenen ist bei Kindern eher seltener, wird aber in der Regel nicht auf Volumen alleine ansprechen. Hier ist vor einer alleinigen Gabe zu großer Flüssigkeitsmengen bei warmer, weitgestellter Peripherie zu warnen, ohne Vasopressoren ist hier meist keine Stabilisierung möglich.

Peripherer Widerstandsverlust

Eine Gabe von Vasokonstriktoren ist indiziert wenn der Patient peripher warm und gut perfundiert erscheint, jedoch kein ausreichender Blutdruck erreicht wird. Hierbei sollte im Röntgen-Thorax das Herz nicht groß erscheinen, der ZVD $<12-15 \mathrm{~cm}$ $\mathrm{H}_{2} \mathrm{O}$ sein. Man wählt in der Pädiatrie traditionell Dopamin (5-) $15-25 \mu \mathrm{g} / \mathrm{kg} / \mathrm{min}$ (> $10 \mu \mathrm{g} / \mathrm{kg} / \mathrm{min}$ : nur zentralvenös), welches. ab ca. $10-15 \mu \mathrm{g} / \mathrm{kg} / \mathrm{min}$ durch eine endogene Noradrenalinausschüttung einen Effekt auf die peripheren alpha-adrenergen Rezeptoren ausübt. Allerdings sind Kinder <6 Monaten wegen mangelnder Freisetzungsfähigkeit von Noradrenalin durch Dopamin teilweise refraktär [55]. Statt Dopamin kann gleich initial oder spätestens wenn der Dopamin-Effekt nicht ausreicht, sekundär Noradrenalin 0,05-1,5 $\mathrm{\mu g} / \mathrm{kg} / \mathrm{min}$ infundieren. Eine kreislaufbedingte Oligurie ist keine Kontraindikation. Ob im Falle eines Versagens der vasokonstriktorischen Wirkung des Noradrenalins (warm shock) neben einer ausreichenden Steroidsubstitution und ggf. Korrektur einer extremen metabolischen Azidose die Gabe von Vasopressin oder eines seiner Analoga prognostisch vorteilhaft sein könnte, ist nicht endgültig gesichert [7, 10, $53,58,77]$. Immer ist auf eine ausreichende Flüssigkeitssubstitution zu achten.

\section{Verminderte Myokardkontraktilität}

Neuere Untersuchungen unter Einsatz von molekulargenetischen Methoden deuten zumindest bei der Sepsis durch Meningokokken darauf hin, dass einer der lange gesuchten Myokarddepressiven Faktoren bei Sepsis von peripheren Leukozyten gebildetes IL6 sein könnte [56]. Ob dies auch unter den Umständen einer Leukopenie zutreffen kann, ist allerdings zumindest zweifelhaft.

Typisch ist ein vergrößertes Herz und/oder erhöhter ZVD. Die Diagnose kann mittels Herzultraschall wahrscheinlich gemacht werden; wegen des kleinen Thorax bei Kindern reicht die 
Eindringtiefe des Ultraschalles zur Beurteilung aus, so dass eine transösophageale Darstellung meist entbehrlich ist. Zur Verwendung der Ergebnisse des Herz-Ultraschalls in dieser klinischen Situation liegen jedoch derzeit wegen mangelnder Erfahrung bei Kindern noch keine Empfehlungen vor [27].

Initial gibt man Dobutamin 5-15(-20) $\mu \mathrm{g} / \mathrm{kg} / \mathrm{min}$. Manchmal resultiert daraus eine periphere Vasodilatation mit Tachykardie und Blutdruck-Abfall, so dass zusätzlich Dopamin oder Noradrenalin erforderlich sind. Bei mangelhaftem Systemwiderstand kann Vasopressin $(1-3 \mathrm{mU} / \mathrm{kg} / \mathrm{min})$ oder Terlipressin $(14 \mu \mathrm{g} / \mathrm{kg}$ in $2 \mathrm{ED}$ ) versucht werden. Bei nicht ausreichendem positiv inotropem Effekt ist die Gabe von Adrenalin $(0,1-2 \mu \mathrm{g} / \mathrm{kg} / \mathrm{min})$ oft erfolgreich.

Bei enggestellter Peripherie mit hohem Systemwiderstand (cold shock) trotz ausreichendem Volumen-Substitution (d. h. gut gefüllten Venen, eher hohem ZVD) und niedrigem Herzzeitvolumen kann (bei normalisiertem Blutdruck!) die zusätzliche Gabe von nachlastsenkenden, lusiotropen Phophodiestersehemmern wie Milrinon $(0,5-0,75 \mu \mathrm{g} / \mathrm{kg} / \mathrm{min})$ sinnvoll sein [10,62]. Andere Autoren empfehlen wegen der kurzen Halbwertszeiten einen initialen Versuch mit Nitroglyzerin (oder Nitroprussid) [28], und einen Übergang zu Milrinon nur bei Zyanid/MethämoglobinToxizität [27]. Bei jeder Vasodilatatortherapie kann die Gabe von zusätzlichen Volumenboli notwendig sein, wenn der Blutdruck in der Folge zu stark absinkt.

Bleibt der Patient trotz maximaler Adrenergika-Gabe und guter Vorlast arteriell hypotensiv und zentralisiert, kann die Anwendung von ECMO (extrakorporaler Membranoxygenierung) erwogen werden. Die Überlebensraten in dieser Situation liegen bei $37-50 \%[9]$.

\section{Sonstige medikamentöse Therapie Steroide}

Hydrokortison in Stressdosis ist zumindest beim adrenergikaresistenten Schock und bei Patienten mit Risikofaktoren für eine NNR-Dysfunktion (Vortherapie mit Steroiden, ZNS-Erkrankungen mit Störungen der Hypophysenregion, Purpura, NNR-Blutung) eventuell lebensrettend [20,27]. Mindestens einer dieser Risikofaktoren liegt bei Kindern mit onkologischen Grunderkrankungen besonders häufig vor. Die richtige Dosis ist unklar, empfohlen wird die Gabe von bis zu $50 \mathrm{mg} / \mathrm{kg}$ im Schock, gefolgt von einer Infusion der gleichen Menge/24 Std [69]. Glukokortikoide in Hochdosis haben sich nicht als sinnvoll erwiesen.

\section{Aktiviertes Protein C}

Protein C hat sowohl gerinnungs- als auch entzündungshemmende Eigenschaften. Bei Erwachsenen mit septischem Schock hat die Gabe von aktiviertem Protein C zu einer höheren Überlebensrate besonders bei Patienten mit einem hohen Mortalitätsrisiko geführt, allerdings war in dieser Studie die Mortalität bei Patienten mit einem geschätzten Sterbe-Risiko $<10 \%$ bei der Verum-Gruppe erhöht [14]. Zusätzlich war eine erhöhte Hirnblutungsrate beobachtet worden. Das Medikament wurde für Erwachsene mit einer erwarteten Mortalität $>20 \%$ zugelassen. Bei Erwachsenen wird die Gabe bei APACHE (Acute Physiology And Chronic Health Evaluation) II-Scores $>25$ ohne Kontraindikation (insbesondere erhöhtes Blutungsrisiko, vorherige oder gleich- zeitige Therapie mit Gerinnungshemmern etc.) empfohlen [32]. Die Pädiatrische Phase-III-Studie wurde nach der Interimsanalyse wegen des ungünstigen Nutzen-Risiko-Profils eingestellt (http://pedsccm.wustl.edu/clinical_research.html\#announce; accessed 18.4.2005).

\section{Besonderheiten bei Kindern mit onkologischen Grunderkrankungen}

Onkologische Patienten mit septischem Schock bieten einige Besonderheiten, die für die Therapie äußerst relevant sind. Ohne die Überwindung der dem septischen Schock zugrunde liegenden Infektion kann kein Patient überleben. Diese kann einerseits die Sanierung eines Fokus der Infektion (z. B. intraabdominelle Herde, Darmperforationen, Abszesse, ggf. nicht durch über die Lumina wechselnd infundierte Antibiotika sanierbare zentralvenöse Katheter) erforderlich machen, andererseits muss eine eventuell bestehende Immunsuppression (auch nach KMT) so rasch als möglich unterbrochen werden. Immunglobuline müssen bei erniedrigten Spiegeln ersetzt werden. Die Gabe von Wachstumsfaktoren beim Granulozytopenischen Kind mit Sepsis kann zu einer rascheren Erholung von der Granulozytopenie führen, hier existieren für Erwachsene eindeutige Empfehlungen [32].

\section{Supportive Intensiv-Therapie}

Ein akutes Nierenversagen ist in der initialen Phase des septischen Schockes häufig die Folge eines zu niedrigen Blutdruckes und ggf. bestehender Elektrolytverschiebungen. Gelingt es, die Perfusion wieder herzustellen und den arteriellen Druck ausreichend anzuheben, ist es meist reversibel. Die Anwendung von Furosemid, Chlorothiazid und Theophyllin (angestrebter Spiegel: 2-4 mg/dl) kann bei drohender Flüssigkeitsüberladung und eingeschränkter Nierenleistung ausreichen [11]. Bei im Gefolge des Multiorganversagens bei Sepsis auftretendem Nierenversagen durch Tubulusnekrose hat sich die Anwendung der kontinuierlichen veno-venösen Hämofiltration bewährt [24, 26, 44].

Beim beatmeten Kind muss eine Analgosedierung, z.B. mit Fentanyl (3-5 $\mu \mathrm{g} / \mathrm{kg} / \mathrm{Std}$.), Morphin oder Ketamin sowie Midazolam $(0,1-0,3 \mathrm{mg} / \mathrm{kg} / \mathrm{Std})$ bzw. Diazepam erfolgen, um Stress und Energieverbrauch zu mindern.

Der Hb-Wert soll $>10 \mathrm{~g} / \mathrm{dl}$ gehalten werden, um ein ausreichendes $\mathrm{O}_{2}$-Angebot in den Zielorganen zu ermöglichen [63].

Die Glukosezufuhr soll bei Kindern unter 10 Jahren $6-8 \mathrm{mg} / \mathrm{kg} /$ min betragen; bei älteren Patienten $4-5 \mathrm{mg} / \mathrm{kg} / \mathrm{min}$, eine Korrektur der Dosis erfolgt nach Blutzuckerkontrollen (möglichst keine Hyperglykämie zulassen). Neuere Daten, die allerdings von Erwachsenen mit chirurgischen Grunderkrankungen stammen, haben eine verminderte Inzidenz von Pilzinfektionen und ein verbessertes Überleben gezeigt, wenn der Blutzucker streng kontrolliert wird und auch geringe Hyperglykämien durch Insulingabe strikt vermieden werden [75]. Ob dies allerdings auch für Kinder mit onkologischen Grunderkrankungen und Sepsis so gilt, ist nicht erwiesen, eine bei diesem Vorgehen besonders leicht eintretende Hypoglykämie ist gerade hier unerwünscht. Eine Hyperglykämie über $200 \mathrm{mg} / \mathrm{dl}$ sollte jedoch nicht akzeptiert werden. 
Die Kalorienzufuhr sollte bei Kindern unter $10 \mathrm{~kg} 55-75 \mathrm{kcal} / \mathrm{kg} /$ Tag, bei älteren Patienten $45-55 \mathrm{kcal} / \mathrm{kg} / \mathrm{Tag}$ nicht unterschreiten. Wegen der Gefahr von Magenblutungen und Stressulzera gibt man bei einem Magensaft-pH <4,5 Magensäureblocker, z.B. Ranitidin $5 \mathrm{mg} / \mathrm{kg} / \mathrm{Tag}$ in $2 \mathrm{ED}$ bzw. Omeprazol. Dennoch deuten pädiatrische Studien darauf hin, dass bei nicht-onkologischen Kindern keine wirkliche Indikation zur Säureblockade besteht, da fast nie bedrohliche Blutungen auftreten. Dies könnte jedoch bei Kindern mit Thrombopenie anders sein.

Sobald als möglich (meist nach 24-72 Stunden) soll mit einer oralen Zufuhr, z.B. von 5\%iger Glukose ( $1 \mathrm{ml} / \mathrm{kg}$ alle 4 Stunden) begonnen werden. Später erfolgt der Übergang auf ein isoosmolares Hydrolysat, sonst bzw. zusätzlich wird die parenterale Ernährung aufgebaut. Dabei werden Lipide nur bei Serum-Triglyzeridspiegel $<80 \mathrm{mg} / \mathrm{dl}$ gegeben.

\section{Ausblick}

Eine Fülle von additiven Therapiemaßnahmen ist beim septischen Schock vorgeschlagen oder versucht worden. Negativ verlaufene Therapie-Studien wurden mit ATIII, monoklonalen Antikörpern gegen Endotoxin (HA-1A, E5), anti-TNF-Antikörpern, löslichem TNF-Rezeptor, Anti-Bradykinin, IL-1-Rezeptor-Antagonisten, PAF-Antagonisten und Ibuprofen unternommen.

Bei Erwachsenen mit septischem Schock wird dezidiert von der Gabe von AT III, Hochdosis-Steroiden (>300 mg Hydrokortison/ Tag) und FFP (Ausnahme: relevante Blutung bzw. OP-Vorbereitung) wegen negativ verlaufener Studien (z.T. mit Erhöhung der Mortalität) abgeraten [29]. Thrombozytenkonzentrate werden bei Erwachsenen ohne manifeste Blutung erst bei Werten ab

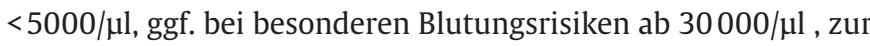
OP-Vorbereitung und bei Blutungen auch darüber, empfohlen.

Tab. 2 Initiale Schock-Therapie

1. großlumiger Zugang ( $20 \mathrm{~g}<15 \mathrm{~kg}, 18 \mathrm{~g}>15 \mathrm{~kg}$ ), ggf. introssäre Nadel

2. Volumen

(„aus der Hand“, wdh. Boli a $20 \mathrm{ml} / \mathrm{kg}$ Vollelektrolytlösung (20-40 ml/kg/5-10 Min.)

dann z. B. $20 \mathrm{ml} / \mathrm{kg} \mathrm{HA} 5 \%$ (später ggf. FFP), Gesamtvolumen (1. Std. ) bis zu $100(-200) \mathrm{ml} / \mathrm{kg}$, bzw. bis ausreichende Zirkulation wiederhergestellt (Maximum: Halsvenen bei 30 Grad gefüllt bzw. ZVD 12-15), EK bei $\mathrm{Hb}<8 \mathrm{~g} / \mathrm{dl}$; FFP bestellen und TK bereitstellen lassen

3. Antibiotika

4. vasoaktive Medikation

Noradrenalin $(0,05-1,5 \mu \mathrm{g} / \mathrm{kg} / \mathrm{min})$ o. Dopamin $(5-25 \mu \mathrm{g} / \mathrm{kg} / \mathrm{min})$, bei volumenrefraktärer Hypotension (Koronarperfusion!) Dobutamin $(5-20 \mu \mathrm{g} / \mathrm{kg} / \mathrm{min})$ bzw. Adrenalin $(0,05-1 \mu \mathrm{g} / \mathrm{kg} / \mathrm{min})$ bei V.a. myokardiale Depression

5. Intubation

evtl. aber auch erste Maßnahme! (oral; bei Hypotension z. B. mit Ketanest oder Hypnomidate einleiten) bei

1. refraktärem Schock

2. Sättigung unter $90 \%$ unter $21 \mathrm{O}_{2}$-Vorlage

3. Bewusstseinstrübung

6. Hydrokortison

z. B. $2 \mathrm{mg} / \mathrm{kg}$ i.v. (evtl. bis $50 \mathrm{mg} / \mathrm{kg}$ ), gefolgt von einer Dauerinfusion

in derselben $(2 \mathrm{mg} / \mathrm{kg})$ Dosis $/ 24$ Std.
Obwohl nicht klar ist, ob sich diese Empfehlungen auch auf Kinder übertragen lassen (z. B. raschere ATIII- und Protein C-Depletion bei Kindern [31, 32], wobei allerdings eine ATIII-Gabe bei Kindern mit septischem Schock erfolglos blieb, ist zumindest eine routinemäßige Anwendung von FFP, ATIII und Protein C [31] zweifelhaft und kann derzeit außerhalb von Studien noch nicht empfohlen werden. Bei gemessen erniedrigten Spiegeln und protrahierter intravasaler Gerinnung kann im Einzelfall anders entschieden werden.

Als adjunkte Maßnahmen, über deren Wert zum Teil nichts Abschließendes bekannt ist, die aber im Einzelfall erwogen werden können, wurden unter anderem vorgeschlagen Heparin, Urokinase, C1-Esterase-Inhibitor, Protein C, rTPA, Hämofiltration, eine Granulozytentransfusion, Immunglobuline trotz normaler Serumwerte, sowie die Gabe von Naloxon und Thyroxin.

Die Anwendung dieser Therapien sollte strikt Studien vorbehalten bleiben.

\section{Akutes Lungenversagen}

\section{Allgemeines (Ursache, Definition, Prognose) Definition}

Ein akutes Lungenversagen kann durch direkte Lungenschädigung, d.h. Einwirkung der Noxe auf die Alveozyten (z.B. bei Rauchgasinhalation) oder indirekt, d.h. durch Schädigung der Lungenkapillaren (z. B. Sepsis) hervorgerufen werden. Die derzeit am häufigsten verwendete, von einer Amerikanisch-Europäischen Konsensuskonferenz erstellte Definition des Lungenversagens [12] benutzt den Oberbegriff „Akutes Lungenversagen“ („Acute Lung Injury [ALI]) für jede akute, alveoläre Erkrankung, die zu einer Störung der Oxygenierung mit einem Abfall des $\mathrm{paO}_{2} /$ $\mathrm{FiO}_{2}$-Quotienten $<300 \mathrm{mmHg}$ führt. Als Akutes Atemnotsyndrom (ARDS) wird eine Subgruppe der Patienten mit nicht kardiogenem Lungenödem (pulmonalkapillärer Wedgedruck $<18 \mathrm{~mm} \mathrm{Hg}$, falls gemessen), bilateralen alveolären Infiltraten im Röntgenbild, und einem $\mathrm{paO}_{2} / \mathrm{FiO}_{2}$-Quotienten $<200 \mathrm{~mm} \mathrm{Hg}$ bezeichnet. Die Definition ist strittig, da der Quotient durch Beatmung zu beeinflussen ist, aber die Beatmungsbedingungen unter denen der Parameter erhoben wurde nicht berücksichtigt werden.

\section{Inzidenz}

Die jährliche alters- und populationsbezogene Inzidenz des ARDS bei Kindern jenseits der Neugeborenenperiode und Jugendlichen liegt bei 3-6/100 000 Einwohnern dieser Altersgruppe [17]. Etwa dreißig Prozent der Kinder leiden unter einer Störung des Immunsystems. Somit treten jährlich ca. 100-200 Fälle eines Lungenversagens bei immunsupprimierten Kindern und Jugendlichen in Deutschland auf. Unter den intensivpflegebedürftigen hämatologisch-onkologischen Patienten leiden ca. $25 \%$ unter einer Lungenparenchymerkrankung, etwa $15 \%$ sind intubationspflichtig [40].

\section{Ursachen}

Häufigste Ursachen bei immunsupprimierten Patienten sind Pneumonien durch meist opportunistische Erreger und Pneumonien ohne Erregernachweis (Graft-vs-Host-Reaktionen, Engraftment-Syndrom, medikamenteninduzierte Pneumonien, nicht 
identifizierte Infektionserreger). Etwa 25\% der Fälle werden durch eine bakterielle Sepsis hervorgerufen.

\section{Prognose}

Eine Störung der Immunabwehr stellt den wichtigsten unabhängigen Risikofaktor für das Überleben des ARDS dar [23]. In den vergangenen zehn Jahren ist eine deutliche Besserung der Prognose eingetreten. Daten aus der ARDS-Datenbank, die seit 1991 Ursache und Ausgang des ARDS von über 400 Kindern gesammelt hat, zeigen einen Rückgang der Sterblichkeit immunsupprimierter Patienten ( $n=134$ ) von 77\% im Zeitraum 1991-1993 auf $42 \%$ im Zeitraum 1997-2000. Dies deckt sich mit von Hallahan et al. berichteten $48 \%$ [40]. Einige unizentrische Daten zeigen z.T. noch günstigere Prognosen [43]. Besonders hoch ist jedoch die Letalität des Lungenversagens nach KMT. Die ARDS-Datenbank erfasste nach 1995 fünfzehn solcher Kinder mit einer Letalität für die Intensivtherapie von 83\% [61\%, 94\%]. Tomaske et al. [74] berichten eine Letalität von $85 \%$ für beatmungspflichtige pädiatrische KMT-Patienten. Allerdings werden auch bessere Überlebensraten in dieser Gruppe berichtet [65].

\section{Pathophysiologie und klinische Symptome}

Die Läsion der alveolokapillären Membran verursacht eine schwere Diffusionsstörung, die sich klinisch durch Tachypnö und stöhnende Atmung, anfangs evtl. begleitet von einer respiratorischen Alkalose äußert. Die alveolokapilläre Läsion bzw. der ursächliche oder sekundär entstehende Entzündungsprozess hat die Leckage von Proteinen in den Alveolarraum zur Folge. Der Proteinübertritt verhindert auch bei normalem Kapillardruck die Rückresorption von Flüssigkeit aus dem interstitiellen Lungengewebe und den Alveolen (Permeabilitätsödem). Bei der Intubation kann fleischwasserfarbene Ödemflüssigkeit aus dem Tubus austreten. Durch die intraalveoläre Proteinansammlung, durch Entzündungsvorgänge und durch die Zellschädigung der Pneumozyten II kommt es zu einem sekundären Verlust an Surfactant. Die surfactantabhängige Verminderung der Oberflächenspannung und damit die Eröffnung der betroffenen Alveolarbezirke bei relativ niedrigen Drücken, geht verloren. Ein Absinken des exspiratorischen Druckes unter diesen so erhöhten Verschlussdruck (s. Abb. 2, Punkt B) führt zum Alveolarkollaps und erfordert hohe Inspirationsdrücke, um verschlossene Bezirke wieder zu eröffnen. Mit der Ausbildung von Atelektasen kommt es so zu einer erheblichen Minderung der funktioneller Residualkapazität, die Lungencompliance nimmt drastisch ab. Zusätzlich kommt es zu einem ausgeprägtem intrapulmonalem Shunt, d.h. einem Missverhältnis von Ventilation und Perfusion (VA/Q). Nur durch einen ausreichend hohen endexspiratorischen Druck (PEEP), der oberhalb des exspiratorischen Wendepunktes der Druckvolumen-Kurve liegt (s. Abb. 2, Punkt B), kann der weitere Kollaps von Alveolen vermindert werden. Klinisch ist die akute Phase durch die Entwicklung von Dyspnö und hohem Sauerstoffbedarf gekennzeichnet. Dieses Vollbild entwickelt sich oft nach einer Latenzphase von 6-72 Stunden. Bei Patienten in der Aplasie kommt es häufiger erst mit Erholung aus der Granulozytopenie zu einer kritischen Verschlechterung der Ventilation [54]. Die prolongierte Anwendung von GCSF scheint die Entwicklung eines ARDS zu begünstigen [73].

Das interstitielle Ödem und das alveoläre Permeabilitätsödem und der begleitende Entzündungsprozess bestimmen den Ver- lauf der ersten Krankheitstage (exsudative Phase). Die Lungenschädigung ist zwar diffus, die sekundären Effekte betreffen aber nicht homogen alle Lungenabschnitte. Vor allem abhängige Partien (s. Abb. 3) sind stärker vom Ödem betroffen [64]. In dieser akuten Phase ist die Lungenfunktionsstörung rein restriktiv, auskultatorisch finden sich ohrnahe fein- bis mittelblasige Ras-

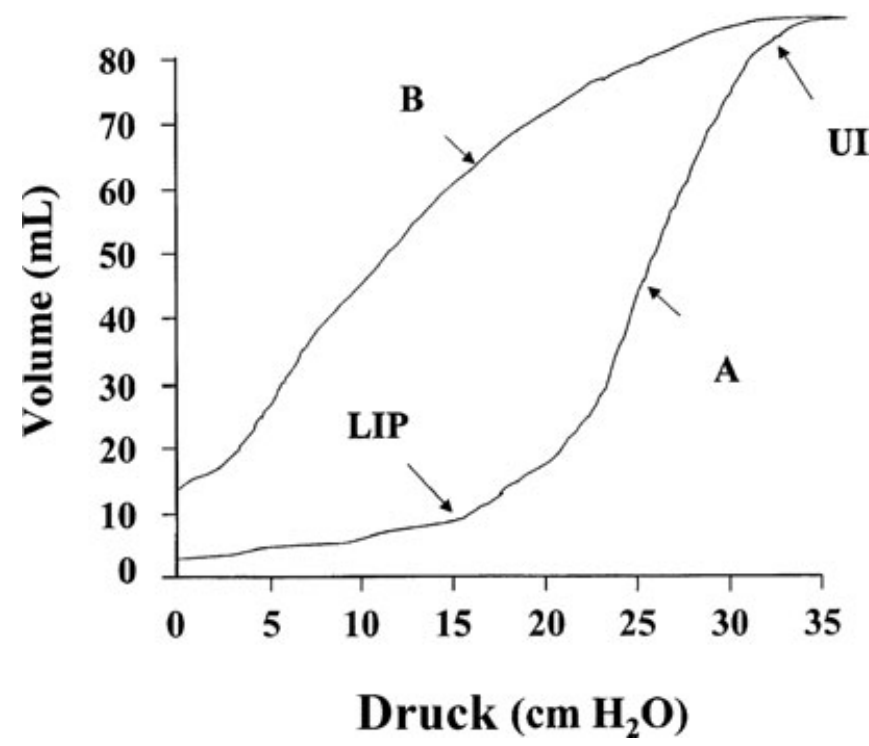

Abb. 2 Druck-Volumenkurve.
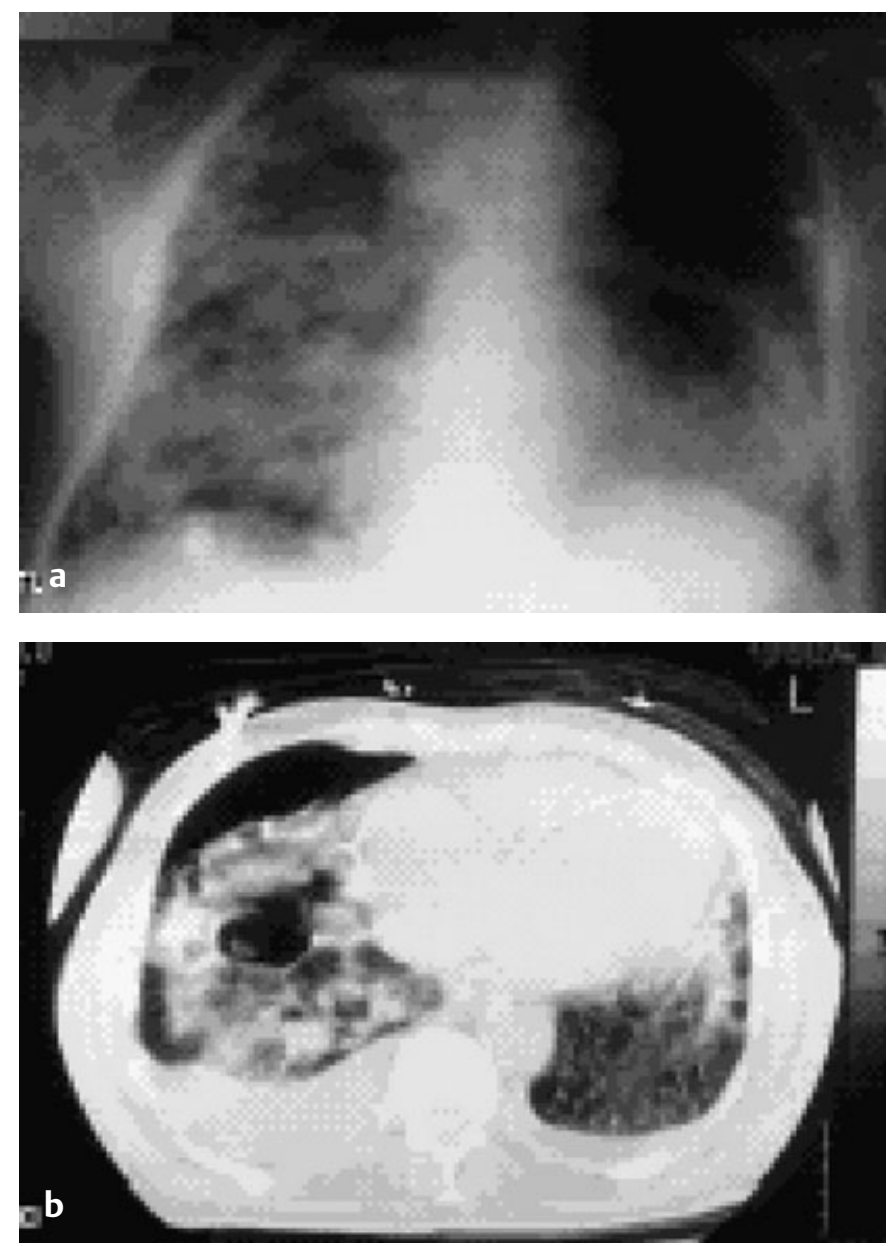

Abb. 3 Atelektasen in abhängigen Partien 
selgeräusche, das Exspirium ist nicht verlängert. Zur primären Noxe kommen mehr oder weniger ausgeprägte sekundäre, d.h. therapiebedingte Schäden hinzu. Die Beatmung mit für den geringen Anteil noch funktionstüchtiger Lungenbezirke inadäquat hohen Tidalvolumina, führt zum Auftreten von Scherkräften und zur Überdehnung dieser noch intakten Regionen. Diese Beatmungsfolgen („ventilator induced lung injury“) und oxidativer Stress tragen zur weiteren Destruktion der alveolokapillären Membran bei. „For more than a quarter of a century the attainment of normal blood gases has been the target of ventilatory management of ARDS. Recent experimental and clinical evidence has shown, that the achievement of this therapeutic goal has an unfavourable cost/benefit ratio, and that ARDS, as we know it, might be more the product of our therapy than of the natural history of the underlying disease“ [57]. Sekundäre Pneumonien und Überwässerung können die Lungenschädigung verschlimmern. Das Lungengewebe wird durch mechanisch induzierte Entzündungsvorgänge selbst zum Herd einer systemischen Entzündungsreaktion (SIRS) [61, 68, 70].

Gelingt es, die auslösende Noxe frühzeitig auszuschalten und die genannten Komplikationen zu vermeiden, so kommt es bei intakten Reparaturvorgängen bereits in den ersten Krankheitstagen zur Wiederherstellung der alveolokapillären Membran und Resorption der interstitiellen und alveolären Flüssigkeit sowie zur Proliferation von Pneumozyten II. Die Reparatur der extrazellulären Matrix erfordert die balancierte Interaktion eines diffizilen Netzwerkes von Entzündungsmediatoren, Makrophagen und Wachstumsfaktoren sowie eine ausreichende Zahl residueller Pneumozyten $[15,50]$. Das Verständnis dieses Prozesses ist noch zu gering, um gezielte medikamentöse Interventionen zu erlauben.

Kommt es nicht zu einer frühzeitigen Reparatur der alveolokapillären Grenzschicht, so geht die Erkrankung nach ein bis zwei Wochen in eine fibroproliferative Phase mit restriktiver und obstruktiver Lungenfunktionsstörung über oder nimmt einen fulminanten hypoxämischen Verlauf [6].

Zu der Störung der Oxygenierung selbst kommt eine Beeinträchtigung der Systemzirkulation. Die hypoxische pulmonale Vasokonstriktion bei Minderbelüftung und die entzündlichen bzw. mikrothrombotischen Veränderungen der Lungenstrombahn verursachen einen Anstieg des Lungengefäßwiderstandes, der zur Rechtsherzinsuffizienz führen kann. Der hohe Atemwegsmitteldruck beeinträchtigt zusätzlich den systemvenösen Rückstrom. Wegen der Steifigkeit des Lungenparenchyms wird der hohe Beatmungsdruck allerdings nur teilweise an das Gefäßsystem weitergeleitet. Die Störung des Rückstroms wird weniger durch eine direkte Kompression der thorakalen Vena cava als durch eine Beeinträchtigung der diastolischen Ventrikelfüllung verursacht. Nur unter differenzierter Kreislauftherapie gelingt es, eine ausreichende Vorlast (intrathorakales Blutvolumen) zur Füllung der Ventrikel aufrecht zu erhalten und andererseits eine Verminderung des freien Lungenwassers und damit eine verbesserte Diffusion von Sauerstoff herbeizuführen.

\section{Diagnostik}

Als bildgebendes Verfahren zur Beurteilung der Ausdehnung der Infiltrate und Lagekontrolle der Installationen ist die a.p. Röntgenthoraxaufnahme ausreichend. Ein $\mathrm{CT}$ ist nur bei V.a. eine As- pergillose oder zur Fokussuche bei schwerer Sepsis indiziert. Hinsichtlich der Ursachendiagnostik sei auf den entsprechenden Beitrag in diesem Heft verwiesen.

Die apparative Überwachung des Patienten muss neben der blutigen Messung des arteriellen Blutdrucks und der arteriellen Blutgase die kontinuierlichen Messung der Diurese und die intermittierende Messung der zentralvenösen Sauerstoffsättigung umfassen sowie die kontinuierliche Messung des ZVD, der indirekt einen Anstieg des Lungengefäßwiderstandes reflektieren kann. Die invasive Messung des pulmonalarteriellen Druckes und des Wedge-Druckes ist nicht erforderlich. Eine ausgeprägte Rechtsherzbelastung kann über einen Anstieg des ZVD und die Echokardiographie festgestellt werden. Als neue, weniger invasive Alternative zur pulmonalarteriellen Thermodilutionsmessung des Herzzeitvolumens, des intrathorakalen Blutvolumens und des freien Lungenwassers steht die PICCO-Technik zur Verfügung, die auch bei Säuglingen anwendbar ist. Allerdings ist die Gültigkeit der üblichen Referenzwerte für Kinder fraglich und die Messmethode ist mit einem hohen Fehlerrisiko (z.B. durch Pleuraergüsse oder intrakardiale Shunts) behaftet.

Die Schwere des Lungenversagens kann neben dem $\mathrm{paO}_{2} / \mathrm{FiO}_{2}$-Quotienten durch den Oxygenierungsindex

$\mathrm{OI}=$ Atemwegsmitteldruck $\left[\mathrm{cmH}_{2} \mathrm{O}\right] \times 100 \times \mathrm{FiO}_{2} / \mathrm{p}_{\mathrm{a}} \mathrm{O}_{2}$

beschrieben werden.

\section{Therapiemaßnahmen}

Die wichtigsten Elemente der Therapie des akuten Lungenversagens sind:

- Elimination der Noxe

- Lungenprotektive Beatmung (Minderung von Scherkräften durch zu hohe Atemzugvolumina und mangelnden PEEP, Verhinderung des Alveolarkollaps durch PEEP)

- Differenzierte Kreislauftherapie (Aufrechterhaltung eines ausreichenden Herzzeitvolumens durch adäquate Preload, Behandlung einer hämodynamisch relevanten pulmonalen Hypertension und inotrope Behandlung einer evtl. Myokarddysfunktion; Reduktion des Lungenwassers)

\section{Evidenzbasierte Therapieempfehlungen}

Eine Fallserie und eine kontrollierte Studie [42] weisen auf eine geringere Letalität immunsupprimierter erwachsener Patienten hin, bei denen zugunsten einer Maskenbeatmung auf eine Intubation verzichtet wurde. Dies lässt eine Stufe C-Empfehlung für den Versuch einer nichtinvasiven Beatmung zu, die allerdings bei kleineren Kindern oft auf Kooperationsprobleme trifft.

Für die Beatmungstherapie ist die Begrenzung der Atemzugvolumina $(<7 \mathrm{ml} / \mathrm{kg}$ ) unter Akzeptanz erhöhter KohlendioxidPartialdrücke („permissive Hyperkapnie“) auf Stufe A nach der DELPHI-Klassifikation durch fünf randomisierte, kontrollierte Studien an Erwachsenen [1, 3, 21, 22, 67] gesichert. Dieser Volumenwert gilt für die tubusnahe Messung und bezieht sich auf das für die Körperlänge zu erwartende Gewicht, nicht auf das tatsächliche Körpergewicht. Die Besserung der Oxygenierung 
durch PEEP wurde bereits in der Erstbeschreibung des ARDS von Ashbaugh 1967 dargestellt [8] und wird durch systematisch erhobene Patientendaten [71] und tierexperimentelle Daten sowie pathophysiologische Überlegungen (s. o.) gestützt. Große randomisierte Studien fehlen, was nur einer Stufe C-Empfehlung entspricht. Der Verzicht auf PEEP ist allein aus den geschilderten pathophysiologischen Überlegungen heraus allerdings indiskutabel. Kontrollierte Studien sind deshalb kaum vertretbar. Ziel ist es, den PEEP knapp oberhalb des unteren Wendepunktes des inspiratorischen Druck-Volumen-Kurve bzw. am Wendepunkt der exspiratorischen Kurve einzustellen, um so, im Bereich der höchsten Compliance eine Beatmung mit minimalen Scherkräften zu ermöglichen. In der pädiatrischen Intensivmedizin ist die Hochfrequenz-Oszillationsbeatmung weit verbreitet. Hier erfolgt der Gasaustausch durch forcierte Diffusion, bei konstant hohem Distensionsdruck der Atemwege über ein CPAP-System. Die Luftsäule wird durch eine Lautsprechermembran in Schwingungen mit einer Frequenz zwischen 3 und $15 \mathrm{~Hz}$ versetzt. Da kein konvektiver Gasaustausch mehr erforderlich ist, können hohe Spitzendrücke vermieden werden. Bei Kindern mit ARDS weist eine kontrollierte Studie [5] darauf hin, dass Beatmungsdauer und Sauerstoffbedarf (Stufe B) verkürzt werden, ein Letalitätsvorteil ist bislang nicht nachgewiesen (Stufe B). Die Anwendung ist technisch nicht ganz unproblematisch und muss Stationen vorbehalten bleiben, die ausreichend Übung mit dieser Beatmungsmethode haben, um Komplikationen rechtzeitig erkennen und beheben zu können.

Durch Bauchlagerung kann der inhomogenen Luft- und Flüssigkeitsverteilung mit stärkerer Minderbelüftung der abhängigen Partien entgegengewirkt werden. Die Verbesserung der Oxygenierung durch überwiegende Bauchlagerung ist durch mehrere Studien sowohl bei Erwachsenen als auch bei Kindern [45] belegt. Bei Erwachsenen mit schwerem Lungenversagen konnte in einer RCT an über 300 Patienten in einer post-hoc-Analyse an 162 schwerst betroffenen Patienten ein Letalitätsvorteil von 20,5 vs. $40 \%$ festgestellt werden [37]. Für die Bauchlage kann eine Stufe B-Empfehlung zur Oxygenierungsverbesserung, jedoch nicht zur Mortalitätsverringerung ausgesprochen werden. Die Bauchlagerung erfordert Vorsichtsmaßnahmen (freie Lage des Abdomens, Schutz vor Dekubitus im Gesicht, cave Tubusdislokation beim Drehen etc.).

Unter den medikamentösen Therapien ist die Gabe von Surfactant durch kleine Fallserien und vier kontrollierte Studien untersucht. Die Behandlung von Erwachsenen mit bovinem Surfactant in einer (open label) Pilotstudie [39] in einer hohen Dosis von $4 \times 100 \mathrm{mg}$ erbrachte einen nicht signifikanten Letalitätsvorteil ( 19 vs. $44 \%$; $p=0,075$ ), während eine Studie mit inhalativem synthetischem Surfactant keinen Vorteil zeigte. Eine offene randomisierte Studie an Kindern [52] zeigte bei kleiner Patientenzahl $(n=35)$ mit schwerem ARDS eine tendenziell geringere Letaltität, jedoch einen signifikant selteneren Einsatz von Rescuetherapien (NO, Vasodilatatoren, HFOV, ECMO) bei Gabe von bovinem Surfactant $(1-2 \times 100 \mathrm{mg} / \mathrm{kg})$. Eine initiale Oxygenierungsverbesserung war nach 48 Stunden nicht mehr signifikant. Die Subgruppe immunsupprimierter Patienten unterschied sich hierin nicht vom Rest. Eine umfangreichere randomisierte Studie an 153 Kindern [78] zeigte eine signifikante Verminderung der Letalität (19 vs. $36 \%$ ). Allerdings waren in der Verumgruppe nur
$28 \%$ der Patienten immundefizient vs. $40 \%$ in der Plazebogruppe. Unter den 55 immundefizienten Patienten war der Letalitätsvorteil (50 vs. $60 \%$ ) nicht signifikant. Bei schwerem ARDS kann somit eine Empfehlung der Stufe B ausgesprochen werden, deren Gültigkeit für hämatologisch-onkologische Patienten aber nicht gesichert ist.

Die einzigen kontrollierten Patientenstudien zur Auswirkung von inhalativem Stickstoffmonoxid [33, 48, 73], welches zu einer Verminderung des Ventilations-Perfusions-Missverhältnisses und zu einem Absinken des Lungengefäßwiderstandes führen kann, haben gezeigt, dass der Oxygenierungsvorteil sich binnen 72 Stunden verliert, die Letalität wird nicht beeinflusst (Stufe A). Die Studien schlossen auch Patienten mit nur mäßig schwerem Lungenversagen ein. In der europäischen Multizenterstudie von Lundin fiel allerdings auf, dass die Studienpatienten mit schwerstem Lungenversagen (arterieller Sauerstoffpartialdruck $<45 \mathrm{mmHg}$ über 2 Stunden bzw. $<60 \mathrm{mmHg}$ für mindestens 6 Stunden) seltener der extrakorporalen Membranoxygenierung (ECMO) zugeführt werden mussten. Bei Kindern mit schwerem ARDS (Oxygenierungsindex $>25$ ) wurde auch über 72 Stunden hinaus in einer kontrollierten Studie häufiger das Erreichen von potenziellen ECMO-Kriterien (OI $>40$ für 3 Stunden) vermieden [35]. ECMO gilt bei immunsupprimierten Patienten als kontraindiziert. Weltweit sind wenig mehr als zehn Patienten mit eingeschränkter Immunkompetenz bzw. hämatologisch-onkologischen Erkrankungen publiziert, die ein ARDS an ECMO überlebt haben. Unter diesem Aspekt kommt der Einsatz von inhalativem Stickstoffmonoxid bei schwerstem ARDS zur Verbesserung der Oxygenierung im Sinne eines individuellen Heilversuches infrage.

Die Gabe hochdosierter Glukokortikoide erhöht die Sterblichkeit des ARDS [13], mehrere kontrollierte Studien lassen eine Stufe-A-Empfehlung zum Verzicht auf hochdosierte Steroide zu. Bei Übergang in eine fibroproliferative Phase kann jedoch eine schnellere Entwöhnung und geringere Letalität laut einer kleineren randomisierten Studie durch Gabe von Prednison $(2 \mathrm{mg} / \mathrm{kg}$ ) erzielt werden [51]. Falls die umfangreichere NHL-LaSRS-Studie, deren Ergebnisse derzeit erwartet werden, die Beobachtung stützt, ergäbe sich eine Stufe B-Empfehlung. Die besondere Situation von hämatologisch-onkologischen Patienten, deren Atemnotsyndrom erst mit Erholung aus der Granulozytopenie auftritt oder sich verschlimmert ist lange bekannt [54]. Der Stellenwert von Steroiden ist hier nicht geklärt. Die Therapie der Pneumocystis-carinii-Infektion und des Engraftment-Syndroms nach Stammzelltransplantation [25] stellen dabei eine Ausnahme dar; hier ist eine Letalitätsminderung durch Gabe von Prednison $(2 \mathrm{mg} / \mathrm{kg})$ bewiesen.

Weitere publizierte pharmakologische Therapieversuche umfassen die Gabe von Ketokonazol, von Ibuprofen, Pentoxiphyllin, liposomalem Prostaglandin E, Antioxidantien (kontrollierte Studien) und die thrombolytische Therapie (Kasuistiken). Die Gabe von Ketokonazol, Pentoxifillin und Ibuprofen haben sich als nicht wirksam, die von liposomalem PGE1 und von Antioxidantien in lediglich je einer Studie als die Beatmungsdauer verkürzend erwiesen. Eine fibrinolytische Therapie ist bei onkologischen Patienten aufgrund der meist bestehenden Thrombozytopenie ohnehin kontraindiziert. In einer Pilotstudie wurde durch frühzeitige venovenöse Hämofiltration eine geringere Letalität 
von Kindern mit ARDS nach KMT berichtet [34]. Eine Evidenzbasierte Empfehlung lässt sich hieraus nicht ableiten.

\section{Praktisches Vorgehen (persönliche Praxis)}

Bei Anstieg des Sauerstoffbedarfes über eine Vorlage von 2 1/min mittels Nasensonde hinaus erfolgt zuerst der Versuch der intermittierenden oder kontinuierlichen Maskenbeatmung (CPAP mit $6-8 \mathrm{~cm} \mathrm{H}_{2} \mathrm{O}$ ). Sinkt die Sättigung trotz eines $\mathrm{FiO}_{2}>0,6$ unter $90 \%$, oder bei klinischer Erschöpfung oder mentalen Zeichen der Dekompensation erfolgt die Intubation. Thrombozytopenische Patienten werden oral intubiert. Die Präoxygenierung erfolgt durch Maskenbeatmung am Ventilator oder unter Blähung mit einem Kuhnsystem, da eine Beutelbeatmung ohne PEEP in dieser Phase zu einer lebensbedrohlichen Hypoxie führen kann.

Die maschinelle Beatmung wird bei nicht-invasiv vorbeatmeten Patienten mit einem $\mathrm{FiO}_{2}$ von 1,0, einem PEEP von $\geq 8 \mathrm{cmH}_{2} \mathrm{O}$ und einem Spitzendruck von $\leq 30 \mathrm{cmH}_{2} \mathrm{O}$ und einem am Tubus gemessenen Tidalvolumen $<8 \mathrm{ml} / \mathrm{kg}$ begonnen. Ist hierunter keine $\mathrm{SaO}_{2}>90 \%$ zu erzielen, wird der PEEP in Inkrementen von $2 \mathrm{~cm}$ $\mathrm{H}_{2} \mathrm{O}$ angehoben und ein Rekrutierungsmanöver (PIP $40 \mathrm{~cm} \mathrm{H}_{2} \mathrm{O}$ im inspiratorischen Hold über 30-40 Sekunden) vorgenommen. Spitzendrücke $>35 \mathrm{~cm} \mathrm{H}_{2} \mathrm{O}$ sollten strikt vermeiden werden. Eine praktische Empfehlung zur Beatmungsführung wurde von der informellen Deutschen Arbeitsgemeinschaft „ARDS im Kindesalter“ [46] publiziert. Bei Beatmung mit niedrigen Tidalvolumina und permissiver Hyperkapnie ist wegen der geringen Reserve der Beatmung ein besonders genaues Monitoring erforderlich, um nicht plötzliche Situationen mit reiner Totraumventilation bei z.B. dyskoordinierter Eigenatmung, Pressen, Sekretobstruktion/Tubusabknickung etc. zu übersehen. Die Beatmung erfolgt mit dezelerierendem Flow, d.h. druckkontrolliert mit einem Inspirationsanteil von $50 \%$ und einer Frequenz die über der altersüblichen Atemfrequenz liegt und mit möglichst wenig Gegenatmung des Patienten verbunden ist. Die Analgosedierung sollte eine Spontanatmung möglichst nicht vollständig unterdrücken, da hierunter ein besseres Ventilations-Perfusions-Verhältnis besteht [60]. Einzelne Patienten entwickeln später im Verlauf der ARDS auch obstruktive Ventilationsstörungen, bzw. bei kleinen Tuben und Sekretakkumulation kann die Anwendung zu kurzer Expirationsphasen zu Auto-PEEP und Kreislaufbzw. Beatmungsproblemen führen. In diesem Fall muss der Exspirationsanteil verlängert werden. Die Lagerung erfolgt überwiegend auf dem Bauch, es empfiehlt sich zur Wirksamkeitsdokumentation eine genaue Analyse der OI-Entwicklung vor und während der Bauchlagenanwendung. In Rückenlage wird der Oberkörper hochgelagert, um das Risiko nosokomialer Pneumonien zu reduzieren. Sind sehr hohe endexspiratorische Drücke $\left(>20 \mathrm{cmH}_{2} \mathrm{O}\right)$ für die Oxygenierung nötig oder trotz Reduktion der Tidalvolumina unter $6 \mathrm{ml} / \mathrm{kg}$ ein Spitzendruck $\geq 30 \mathrm{~cm}$ $\mathrm{H}_{2} \mathrm{O}$ erforderlich, um den Patienten mit einem $\mathrm{FiO}_{2}<0,6$ zu oxygenieren oder um eine schwere respiratorische Azidose mit Rechtsherzbelastung zu vermeiden, so kann ggf. die HFOV eingesetzt werden. Auch ein Versuch mit NO kann zu diesem Zeitpunkt bei einzelnen Patienten mit besonders schweren Verläufen zu signifikanten Besserungen führen (s.u.). Mit Hilfe von Diuretika (Furosemid-DTI, Etacrynsäure) oder nötigenfalls der VVHF wird versucht, das freie Wasser zu vermindern, d.h. eine negative Wasserbilanz zu erzielen.
Eine ausreichende preload, d.h. ein für die Ventrikelfüllung ausreichendes intrathorakales Blutvolumen wird bei hohem freien Lungenwasser mit Erythrozytenkonzentrat bzw. kolloidalen Volumenersatzlösungen erzeugt. Für Kinder sind keine Daten zum Soll-Hb verfügbar, die TRICC-Studie hat bei intensivpflegebedürftigen Erwachsenen keinen Letalitätvorteil durch Transfusion bei $\mathrm{Hb}$-Werten über $7 \mathrm{~g} / \mathrm{dl}$ gefunden. Für die effektive Verwendung von Albumin und Diuretika beim ARDS spricht eine kleine kontrollierte Studie [49]. Die Bedenken gegen kolloidale Lösungen, die aus einer Cochrane-Metananlyse, die in der Subgruppe der ARDS-Patienten nach Trauma eine höhere Letalität bei der Volumensubstitution durch Kolloide gefunden hatte, entstanden sind, konnten bislang nicht belegt werden. Andererseits kann durch Infusion kristalliner Lösungen ein Anstieg des freien Lungenwassers herbeigeführt werden. Zur Steuerung der Volumentherapie kann das PICCO-System, das die Messung des freien Lungenwassers und des intrathorakalen Blutvolumens erlaubt, hilfreich sein. Allerdings setzen seine hohe Anfälligkeit gegen potenzielle Fehlerquellen (Ergüsse, intrakardiale Shunts, technische Probleme) und die mangelnde Übertragbarkeit der Referenzwerte von Erwachsenen hohe Erfahrung voraus.

Die spezifische medikamentöse Therapie umfasst bei uns ferner die Gabe von Prednisolon $(1 \mathrm{mg} / \mathrm{kg}$ ) bei Abklingen der Granulozytopenie, falls nicht bereits wegen einer Sepsis Hydrokortison in Stressdosis verabreicht wird. Entsprechende Studien liegen für diese Praxis nicht vor. Einen Heilversuch mit Inhalation von Stickstoffmonoxid (1-5 ppm) wenden wir bei Abfall des $\mathrm{paO}_{2} /$ $\mathrm{FiO}_{2}$-Quotienten unter $60 \mathrm{mmHg}$ oder vor dem Versuch einer HFOV-Beatmung an, bzw. bei Kreislaufinsuffizienz infolge pulmonaler Hypertension. Bei weiterem Absinken des $\mathrm{paO}_{2} / \mathrm{FiO}_{2-}$ Quotienten erwägen wir in der akuten Phase und schlechter Lungenentfaltung die Applikation exogenen Surfactants $(100 \mathrm{mg} /$ $\mathrm{kg})$.

Bei parenteraler Ernährung werden Fette nur bei Triglyceridwerten $<200 \mathrm{mg} / \mathrm{dl}$ eingesetzt, einer (teil-)enteralen, nötigenfalls postpylorischen Ernährung wird der Vorzug gegeben.

\section{Literatur}

${ }^{1}$ The Acute Respiratory Distress Syndrome Network. Ventilation with lower tidal volumes as compared with traditional tidal volumes for acute lung injury and the acute respiratory distress syndrome. N Engl J Med 2000; 342: $1301-1308$

2 Aledo A, Heller G, Ren L et al. Septicemia and septic shock in pediatric patients: 140 consecutive cases on a pediatric hematology-oncology service. J Pediatr Hematol Oncol 1998; 20: 215-221

${ }^{3}$ Amato MB, Barbas CS, Medeiros DM et al. Effect of a protective-ventilation strategy on mortality in the acute respiratory distress syndrome. N Engl J Med 1998; 338: 347 - 354

${ }^{4}$ Annane D, Sebille V, Charpentier C et al. Effect of treatment with low doses of hydrocortisone and fludrocortisone on mortality in patients with septic shock. Jama 2002; 288: $862-871$

${ }^{5}$ Arnold JH, Hanson JH, Toro-Figuero LO et al. Prospective, randomized comparison of high-frequency oscillatory ventilation and conventional mechanical ventilation in pediatric respiratory failure. Crit Care Med 1994; 22: 1530-1539

${ }^{6}$ Artigas A, Bernard GR, Carlet J et al. The American-European Consensus Conference on ARDS, part 2. Ventilatory, pharmacologic, supportive therapy, study design strategies and issues related to recovery and remodeling. Intensive Care Med 1998; 24: 378-398 
${ }^{7}$ Asfar P. Terlipressin in chronic hyperdynamic endotoxic shock: is it safe? Intensive Care Med 2003; 29: 154-155

${ }^{8}$ Ashbaugh DG, Bigelow DB, Petty TL et al. Acute respiratory distress in adults. Lancet 1967; 2: 319-323

${ }^{9}$ Bartlett RH, Roloff DW, Custer JR et al. Extracorporeal life support: the University of Michigan experience. Jama 2000; 283: 904-908

${ }^{10}$ Barton P, Garcia J, Kouatli A et al. Hemodynamic effects of i.v. milrinone lactate in pediatric patients with septic shock. A prospective, double-blinded, randomized, placebo-controlled, interventional study. Chest 1996; 109: $1302-1312$

${ }^{11}$ Bell M, Jackson E, Mi Z et al. Low-dose theophylline increases urine output in diuretic-dependent critically ill children. Intensive Care Med 1998; 24: 1099-1105

12 Bernard GR, Artigas A, Brigham KL et al. The American-European Consensus Conference on ARDS. Definitions, mechanisms, relevant outcomes, and clinical trial coordination. Am J Respir Crit Care Med 1994; 149: 818 - 824

${ }^{13}$ Bernard GR, Luce JM, Sprung CL et al. High-dose corticosteroids in patients with the adult respiratory distress syndrome. N Engl J Med 1987; 317: 1565 - 1570

${ }^{14}$ Bernard GR, Vincent JL, Laterre PF et al. Efficacy and safety of recombinant human activated protein C for severe sepsis. N Engl J Med 2001; 344: 699-709

${ }^{15}$ Bienkowski RS, Gotkin MG. Control of collagen deposition in mammalian lung. Proc Soc Exp Biol Med 1995; 209: 118 - 140

${ }^{16}$ Bindl L, Buderus S, Dahlem P et al. Gender-based differences in children with sepsis and ARDS: the ESPNIC ARDS Database Group. Intensive Care Med 2003; 29: 1770 - 1773

17 Bindl L, Dresbach K, Lentze MJ. Incidence of acute respiratory distress syndrome in German children and adolescents: a population-based study. Crit Care Med 2005; 33: 209-312

${ }^{18}$ Boldt J, Heesen M, Welters I et al. Does the type of volume therapy influence endothelial-related coagulation in the critically ill? $\mathrm{Br} J \mathrm{An}-$ aesth 1995; 75: 740- 746

${ }^{19}$ Booy R, Habibi P, Nadel S et al. Reduction in case fatality rate from meningococcal disease associated with improved healthcare delivery. Arch Dis Child 2001; 85: 386-390

${ }^{20}$ Briegel J, Forst H, Haller M et al. Stress doses of hydrocortisone reverse hyperdynamic septic shock: a prospective, randomized, double-blind, single-center study. Crit Care Med 1999; 27: 723-732

${ }^{21}$ Brochard L, Roudot-Thoraval F, Roupie E et al. Tidal volume reduction for prevention of ventilator-induced lung injury in acute respiratory distress syndrome. The Multicenter Trail Group on Tidal Volume reduction in ARDS. Am J Respir Crit Care Med 1998; 158: 1831-1838

${ }^{22}$ Brower RG, Shanholtz CB, Fessler HE et al. Prospective, randomized, controlled clinical trial comparing traditional versus reduced tidal volume ventilation in acute respiratory distress syndrome patients. Crit Care Med 1999; 27: 1492 - 1498

${ }^{23}$ Brun-Buisson C, Minelli C, Bertolini G et al. Epidemiology and outcome of acute lung injury in European intensive care units. Results from the ALIVE study. Intensive Care Med 2004; 30: 51 -61

${ }^{24}$ Bunchman TE, Maxvold NJ, Barnett J et al. Pediatric hemofiltration: Normocarb dialysate solution with citrate anticoagulation. Pediatr Nephrol 2002; 17: 150-154

${ }^{25}$ Capizzi SA, Kumar S, Huneke NE et al. Peri-engraftment respiratory distress syndrome during autologous hematopoietic stem cell transplantation. Bone Marrow Transplant 2001; 27: 1299-1303

${ }^{26}$ Carcillo JA. Pediatric septic shock and multiple organ failure. Crit Care Clin 2003; 19: $413-440$

27 Carcillo JA, Fields AI. Clinical practice parameters for hemodynamic support of pediatric and neonatal patients in septic shock. Crit Care Med 2002; 30: 1365-1378

${ }^{28}$ Ceneviva G, Paschall JA, Maffei F et al. Hemodynamic support in fluidrefractory pediatric septic shock. Pediatrics 1998; 102: e 19

${ }^{29}$ Chittock DR, Dhingra VK, Ronco JJ et al. Severity of illness and risk of death associated with pulmonary artery catheter use. Crit Care Med 2004; 32: 911 - 915

30 Cooper DJ, Walley KR, Wiggs BR et al. Bicarbonate does not improve hemodynamics in critically ill patients who have lactic acidosis. A prospective, controlled clinical study. Ann Intern Med 1990; 112: $492-498$

31 de Kleijn ED, de Groot R, Hack CE et al. Activation of protein C following infusion of protein $C$ concentrate in children with severe meningococcal sepsis and purpura fulminans: a randomized, double-blinded, placebo-controlled, dose-finding study. Crit Care Med 2003; 31: 1839 1847

32 Dellinger RP, Carlet JM, Masur $\mathrm{H}$ et al. Surviving Sepsis Campaign guidelines for management of severe sepsis and septic shock. Intensive Care Med 2004; 30: 536-555

${ }^{33}$ Dellinger RP, Zimmerman JL, Taylor RW et al. Effects of inhaled nitric oxide in patients with acute respiratory distress syndrome: results of a randomized phase II trial. Inhaled Nitric Oxide in ARDS Study Group. Crit Care Med 1998; 26: $15-23$

${ }^{34}$ DiCarlo JV, Alexander SR, Agarwal R et al. Continuous veno-venous hemofiltration may improve survival from acute respiratory distress syndrome after bone marrow transplantation or chemotherapy. J Pediatr Hematol Oncol 2003; 25: 801-805

35 Dobyns EL, Cornfield DN, Anas NG et al. Multicenter randomized controlled trial of the effects of inhaled nitric oxide therapy on gas exchange in children with acute hypoxemic respiratory failure. J Pediatr 1999; 134: 406 - 412

${ }^{36}$ Finfer S, Bellomo R, Boyce $\mathrm{N}$ et al. A comparison of albumin and saline for fluid resuscitation in the intensive care unit. N Engl J Med 2004; 350: $2247-2256$

${ }^{37}$ Gattinoni L, Tognoni G, Pesenti A et al. Effect of prone positioning on the survival of patients with acute respiratory failure. $\mathrm{N}$ Engl J Med 2001; 345: $568-573$

${ }^{38}$ Greenhalgh DG, Warden GD. The importance of intra-abdominal pressure measurements in burned children. J Trauma 1994; 36: 685-690

${ }^{39}$ Gregory TJ, Steinberg KP, Spragg R et al. Bovine surfactant therapy for patients with acute respiratory distress syndrome. Am J Respir Crit Care Med 1997; 155: 1309-1315

${ }^{40}$ Hallahan AR, Shaw PJ, Rowell G et al. Improved outcomes of children with malignancy admitted to a pediatric intensive care unit. Crit Care Med 2000; 28: 3718 - 3721

${ }^{41}$ Han YY, Sun WZ. An evidence-based review on the use of corticosteroids in peri-operative and critical care. Acta Anaesthesiol Sin 2002; 40: $71-79$

42 Hilbert G, Gruson D, Vargas F et al. Noninvasive continuous positive airway pressure in neutropenic patients with acute respiratory failure requiring intensive care unit admission. Crit Care Med 2000; 28 : $3185-3190$

43 Jacobe SJ, Hassan A, Veys P et al. Outcome of children requiring admission to an intensive care unit after bone marrow transplantation. Crit Care Med 2003; 31: 1299-1305

${ }^{44}$ Kellum JA, Angus DC, Johnson JP et al. Continuous versus intermittent renal replacement therapy: a meta-analysis. Intensive Care Med 2002; 28: $29-37$

${ }^{45}$ Kornecki A, Frndova H, Coates AL et al. A randomized trial of prolonged prone positioning in children with acute respiratory failure. Chest 2001; 119: $211-218$

${ }^{46}$ Kühl P, Appel R, Lasch P et al. ARDS im Kindesalter. Ergebnisse einer Umfrage an Deutschen Kinderkliniken und Empfehlungen der „Arbeitsgemeinschaft ARDS im Kindesalter" zur Beatmungstherapie. Monatsschr Kinderheilkd 1996; 144: 1110-1116

${ }^{47}$ Larche J, Azoulay E, Fieux F et al. Improved survival of critically ill cancer patients with septic shock. Intensive Care Med 2003; 29: 1688 1695

${ }^{48}$ Lundin S, Mang H, Smithies M et al. Inhalation of nitric oxide in acute lung injury: results of a European multicentre study. The European Study Group of Inhaled Nitric Oxide. Intensive Care Med 1999; 25 : $911-919$

${ }^{49}$ Martin GS, Mangialardi RJ, Wheeler AP et al. Albumin and furosemide therapy in hypoproteinemic patients with acute lung injury. Crit Care Med 2002; 30: 2175-2182

${ }^{50} \mathrm{McGowan}$ SE. Extracellular matrix and the regulation of lung development and repair. Faseb J 1992; 6: 2895 - 2904

${ }^{51}$ Meduri GU, Headley AS, Golden E et al. Effect of prolonged methylprednisolone therapy in unresolving acute respiratory distress syndrome: a randomized controlled trial. Jama 1998; 280: 159-165

52 Moller JC, Schaible T, Roll C et al. Treatment with bovine surfactant in severe acute respiratory distress syndrome in children: a randomized multicenter study. Intensive Care Med 2003; 29: 437-446

53 O'Brien A, Clapp L, Singer M. Terlipressin for norepinephrine-resistant septic shock. Lancet 2002; 359: 1209-1210

54 Ognibene FP, Martin SE, Parker MM et al. Adult respiratory distress syndrome in patients with severe neutropenia. N Engl J Med 1986; 315: $547-551$ 
55 Outwater KM, Treves ST, Lang P et al. Renal and hemodynamic effects of dopamine in infants following cardiac surgery. J Clin Anesth 1990; 2: $253-257$

${ }^{56}$ Pathan N, Hemingway CA, Alizadeh AA et al. Role of interleukin 6 in myocardial dysfunction of meningococcal septic shock. Lancet 2004; 363: 203 - 209

57 Pesenti A. Target blood gases during ARDS ventilatory management. Intensive Care Med 1990; 16: 349-351

${ }^{58}$ Peters MJ, Booth RA, Petros AJ. Terlipressin bolus induces systemic vasoconstriction in septic shock. Pediatr Crit Care Med 2004; 5: 112 - 115

59 Pollard AJ, Britto J, Nadel S et al. Emergency management of meningococcal disease. Arch Dis Child 1999; 80: 290-296

60 Putensen C, Mutz NJ, Putensen-Himmer G et al. Spontaneous breathing during ventilatory support improves ventilation-perfusion distributions in patients with acute respiratory distress syndrome. Am J Respir Crit Care Med 1999; 159: 1241 - 1248

${ }^{61}$ Ranieri VM, Suter PM, Tortorella C et al. Effect of mechanical ventilation on inflammatory mediators in patients with acute respiratory distress syndrome: a randomized controlled trial. Jama 1999; 282: $54-61$

${ }^{62}$ Ringe HI, Varnholt V, Gaedicke G. Cardiac rescue with enoximone in volume and catecholamine refractory septic shock. Pediatr Crit Care Med 2003; 4: $471-475$

${ }^{63}$ Rivers E, Nguyen B, Havstad S et al. Early goal-directed therapy in the treatment of severe sepsis and septic shock. N Engl J Med 2001; 345: $1368-1377$

${ }^{64}$ Rommelsheim K, Lackner K, Westhofen P et al. Respiratory distress syndrome of the adult in the computer tomograph. Anasth Intensivther Notfallmed 1983; 18: 59-64

${ }^{65}$ Rossi R, Shemie SD, Calderwood S. Prognosis of pediatric bone marrow transplant recipients requiring mechanical ventilation. Crit Care Med 1999; 27: 1181 - 1186

${ }^{66}$ Schierhout G, Roberts I. Fluid resuscitation with colloid or crystalloid solutions in critically ill patients: a systematic review of randomised trials. Bmj 1998; 316: 961 - 964
${ }^{67}$ Stewart TE, Meade MO, Cook DJ et al. Evaluation of a ventilation strategy to prevent barotrauma in patients at high risk for acute respiratory distress syndrome. Pressure- and Volume-Limited Ventilation Strategy Group. N Engl J Med 1998; 338: 355 - 361

${ }^{68}$ Stuber F, Wrigge H, Schroeder S et al. Kinetic and reversibility of mechanical ventilation-associated pulmonary and systemic inflammatory response in patients with acute lung injury. Intensive Care Med 2002; 28: $834-841$

${ }^{69}$ Sumarmo. The role of steroids in dengue shock syndrome. Southeast Asian J Trop Med Public Health 1987; 18: 383 - 389

70 Suter PM. MV causes lung inflammation and systemic immune depression. A balance of fire and ice. Intensive Care Med 2002; 28: $383-385$

${ }^{71}$ Suter PM, Fairley B, Isenberg MD. Optimum end-expiratory airway pressure in patients with acute pulmonary failure. N Engl J Med 1975; 292: $284-289$

72 Takahashi Y, Kobayashi Y, Chikayama S et al. Effect of granulocyte/colony-stimulating factor on the onset of the adult respiratory distress syndrome. Acta Haematol 1999; 101: 124-129

73 Taylor RW, Zimmerman JL, Dellinger RP et al. Low-dose inhaled nitric oxide in patients with acute lung injury: a randomized controlled trial. Jama 2004; 291: 1603 - 1609

${ }^{74}$ Tomaske M, Bosk A, Eyrich M et al. Risks of mortality in children admitted to the paediatric intensive care unit after haematopoietic stem cell transplantation. Br J Haematol 2003; 121: 886 - 891

75 den Berghe $G$ van, Wouters P, Weekers F et al. Intensive insulin therapy in the critically ill patients. N Engl J Med 2001; 345: 1359-1367

${ }^{76}$ Watson RS, Carcillo JA, Linde-Zwirble WT et al. The epidemiology of severe sepsis in children in the United States. Am J Respir Crit Care Med 2003; 167: 695-701

77 Westphal M, Bone HG, Aken H Van et al. Terlipressin for haemodynamic support in septic patients: a double-edged sword? Lancet 2002; 360 : $1250-1251$

78 Willson DF, Thomas NJ, Markovitz BP et al. Effect of exogenous surfactant (calfactant) in pediatric acute lung injury: a randomized controlled trial. Jama 2005; 293: 470-476 